\title{
Creación de escenarios ante el aumento del nivel del mar, para las localidades de Moín y Cahuita, Limón, Costa Rica
}

\section{Creation of sea level rise scenarios for the localities of Moín and Cahuita, Limón, Costa Rica}

\author{
Melvin A. Lizano-Araya ${ }^{1}$ \\ Universidad de Costa Rica, Costa Rica
}

Omar G. Lizano-Rodríguez ${ }^{2}$

Universidad de Costa Rica, Costa Rica

\begin{abstract}
Resumen
Se analiza el aumento del nivel del mar tomando como base el nivel promedio de mareas altas para la región Caribe de Costa Rica. Este estudio se realizó a partir de modelos conceptuales, observaciones y mediciones efectuadas en campo, que presentan un nivel del mar y su respectivo ascenso para cuatro escenarios de acuerdo con los siguientes años: 2030, 2050, 2070 y 2100. Se elaboran escenarios para simular el comportamiento del nivel del mar relativo en los próximos 100 años para las localidades de Cahuita y Moín en la provincia de Limón. Se estudian los niveles de mareas astronómicas a partir de predicciones mareales. El aumento del nivel del mar en los próximos años por calentamiento global es, según los escenarios de la CEPAL (2012, p. 24), $3 \mathrm{~mm}$ por año para la costa del Caribe en general y se utiliza como base para crear las distintas combinaciones y generar los niveles mareográficos representados en este artículo. Los resultados muestran los niveles de inundación para cada uno de los años en las localidades de Moín y Cahuita e indica que ambas localidades podrían ser inundadas para el año 2100.
\end{abstract}

Palabras clave: nivel del mar, cambio climático, inundación, topografía, mareas.

1 M.Sc. Docente e investigador, Escuela de Geografía. Universidad de Costa Rica, Costa Rica. E-mail: melvin.lizanoaraya@ucr.ac.cr. (D) https://orcid.org/0000-0003-3437-3502

2 Dr. Docente e investigador, Centro de Investigación en Ciencias del Mar y Limnología (CIMAR) Universidad de Costa Rica, Costa Rica. E-mail: omar.lizano@ucr.ac.cr. (iD https://orcid.org/0000-0001-9407-0322 


\begin{abstract}
Sea level rise is analyzed based on the average level of high tides registered for the Caribbean region of Costa Rica. The present study utilized conceptual models, field observations and field measurements, which provide sea level data and its respective rise, for the modelling of possible scenarios for four different years: 2030, 2050, 2070 and 2100. Scenarios were developed to simulate the behavior of the relative sea level in the next 100 years for the localities of Cahuita and Moín, both in the province of Limón, Costa Rica. Astronomical tidal levels are studied from tidal predictions. The rise in sea level in the coming years due to global warming is, according to ECLAC scenarios/predictions (2012, p. 24), $3 \mathrm{~mm}$ per year for the Caribbean coast in general and is used as a basis for the creation of the different combinations and generation of the tidal levels represented in the present article. Results show flood levels for each considered year and indicate that both Moín and Cahuita could be flooded by the year 2100 .
\end{abstract}

Keywords: Sea level; Climate change; Flood; Topography; Tides.

\title{
Introducción
}

Dentro del Sistema Tierra los efectos de las actividades humanas pueden detectarse a escala planetaria. Los geógrafos físicos han reconocido desde hace tiempo la gran importancia de conocer los rasgos presentes en el terreno a través de los procesos de alteración de las formas del terreno y de los procesos geomorfológicos, incluido el tema de flujos y depósitos hidrológicos hasta los procesos de remoción en masa, erosión y deposición de material en el suelo, los cuales han permitido conocer rasgos antropogénicos para detectar cambios asociados a las actividades humanas realizadas sobre el terreno (Tarolli, Cao, Sofia, Evans \& Ellis, 2019). A lo anterior se le suma que, con base en evidencias que muestran que los procesos atmosféricos, geológicos, hidrológicos y biológicos del Sistema Tierra, entre otros; están siendo alterados por las actividades humanas (PNUMA, 2013, p. XVIII). Los cambios más fácilmente reconocidos incluyen el incremento de las temperaturas globales y de los niveles del mar, así como la acidificación de los océanos asociada al aumento en las emisiones de gases de efecto invernadero (PNUMA, 2013, p. XVIII). Como lo mencionan Dawahidi, Ibarra y Gomariz (2019, p. 40): "El cambio climático producido principalmente por el aumento de las emisiones de Gases de Efecto Invernadero (GEI) generadas por la actividad humana es un fenómeno que ha aumento recientemente debido a otros factores como la explotación de combustibles fósiles, petróleo y carbón".

La extensión de los hielos marinos árticos ha disminuido en 3.5\% y el $4.1 \%$ por decenio, con disminuciones estivales aún más acentuadas 
entre el $9.4 \%$ y el $13.6 \%$ por decenio (IPCC, 2014, p. 44). En promedio, los glaciares de montaña y la cubierta de nieve están decreciendo de manera acelerada, cambio que se ha manifestado en los últimos decenios, a tal punto que es probable que antes de mediados de siglo el océano Ártico esté casi libre de hielo en el mes de septiembre, momento mínimo estival de hielo marino (IPCC, 2014, p. 12). Como lo indica Lambrechts (2007, p. 4): "La extensión de la capa de nieve media mensual en el hemisferio norte ha disminuido a un ritmo d. 1,3\% por decenio en los últimos 40 años, registrándose las mayores pérdidas en la primavera y el verano, lo que supone reducciones en la capa de nieve".

\section{Cambio climático}

Los cambios climáticos reflejan un decremento en las masas glaciares, pero no en todas las regiones se tiene una disminución de la cubierta de nieve. En las regiones del hemisferio norte, los escurrimientos máximos de los glaciares y el derretimiento de la nieve se presentan más temprano en el año provocando la retirada de los glaciares, la fracturación de las grandes plataformas de hielo, el aumento del nivel del mar y el derretimiento del hielo permanente (PNUMA, 2013, p. 118). Todo esto es una influencia directa del calentamiento global, expresado en el informe escrito por el IPCC (2014, p. 10), donde se apunta que es probable que el aumento de la temperatura media global en superficie a final del siglo XXI (2081-2100) respecto de $1986-2005$ sea de $0,3^{\circ} \mathrm{C}$ a $1,7^{\circ} \mathrm{C}$ bajo el escenario RCP 2.6 y donde la región del Ártico seguirá calentándose más rápidamente que la media global. A la vez, el documento indica que es prácticamente seguro que se produzcan temperaturas extremas calientes más frecuentes y frías menos frecuentes en la mayoría de las zonas continentales, en escalas temporales diarias y estacionales, conforme vaya aumentando la temperatura media global en superficie.

A escala global se prevé la inundación de miles de kilómetros cuadrados de humedales costeros y tierras bajas (Bedia, 2004, p. 1). También es previsible el retroceso de playas y la ruptura de infraestructuras litorales de protección, la salinidad de acuíferos y la pérdida de tierras productivas para la agricultura y la ganadería (Bedia, 2004, p. 1). "Los patrones de erosión y sedimentación litorales se verán modificados y afectarán instalaciones portuarias, enclaves turísticos y ecosistemas de especial valor, 
entre otras zonas sensibles" (Van der Meulen, Witter \& Marens, 1991, p. 106). "Las consecuencias previsibles son el retroceso por los cambios en la dinámica litoral, como por la proliferación de estructuras de protección que alterará el ambiente costero en general" (Bedia, 2004, p. 1).

Asimismo, se conoce del último modelo climático, elaborado por Otto-Bliesner et al. (2006, p. 1751) que concuerda con datos obtenidos de registros paleoclimáticos de testigos de hielo, polen fósil, sedimentos marinos y marcas químicas utilizados para comprobar la precisión del modelo. Se efectuó un cálculo del aumento para el año 2100, el cual indica que las costas del sur, suroeste y surestes de los Estado Unidos se verán seriamente afectadas, pues se registra un aumento aproximado de 6 metros del nivel del mar. Ciudades como Nueva Orleans desaparecerán para esa fecha, al igual que ciertas zonas de la Florida, como los cayos (Otto-Bliesner et al., 2006, p. 1752). Otros estudios indican, por ejemplo, que para mediados de siglo los niveles relativos del mar en ciudades como Nueva York, Florida o Miami pueden superar los indicados a inicios del año 2000 en 1.2 m (Wright, Syvitski \& Nichols, 2019). Sin embargo, es importante recalcar que hay otras proyecciones de aumento del nivel del mar que son condicionadas por los RCP y no se ajustan a los diferentes objetivos establecidos en el acuerdo de París donde se pretende mantener el aumento de la temperatura media mundial muy por debajo de los $2^{\circ}$. Los RCP varían en sus cálculos entre un aumento de la temperatura que ronda $\operatorname{los} 1.5^{\circ}$ y $2^{\circ}$ y de ahí las proyecciones de aumento en mayor o menor medida del nivel del mar (Horton et al., 2018).

A nivel mundial, el calentamiento paulatino al que se han visto sometidos los océanos del mundo asociados a las pérdidas de masas de hielo y la expansión térmica causará graves problemas en las áreas costeras, consideradas como uno de los ambientes más frágiles del medio terrestre (Ibarra, Belmonte, Gomariz \& Pérez, 2015). Lo anterior ocasiona pérdidas de tierras habitables y cultivables, intrusión de agua salada y erosión costera (Ibarra-Marinas, 2016).

De igual forma, en la comunidad científica actual hay mucha incertidumbre sobre la respuesta de las grandes capas de hielo de Groenlandia y la Antártida Occidental ante los aumentos de la temperatura de $4^{\circ} \mathrm{C}$ o más para el 2100 y que, eventualmente, situaría el aumento del nivel de los océanos entre los $0.5 \mathrm{~m}$ a $2 \mathrm{~m}$. Consideran que la probabilidad de que se 
produzca un aumento superior es baja, pero no se puede descartar a futuro (Mcinnes, Walsh, Hubbert \& Beer, 2003).

A partir de observaciones satelitales realizadas por los altímetros de la misión TOPEX/Poseidón y las plataformas Jason-1, Jason-2 y Jason-3, estas muestran una aceleración del aumento del nivel de los océanos del mundo con una tasa de incremento de $0.025 \pm 0.084 \mathrm{~mm} / \mathrm{y}^{2}$ para los últimos 25 años y que, al juntarse con la tasa media de aumento del nivel del mar, impulsada por el cambio climático durante estos mismos 25 años, da un promedio de aumento de $2.9 \mathrm{~mm} /$ año (Neremet et al., 2018).

Se debe recalcar que el calentamiento que sufren los océanos se ha distribuido por todo el mundo y no como se ha pensado, que es una distribución localizada, pues varias regiones han mostrado un aumento de la energía térmica presente en las masas de agua (Cheng et al., 2018). Esta energía genera un aumento del nivel del mar por expansión térmica, que unida a otros fenómenos mareográficos como el Niño, apilamiento de oleaje u otros, puede causar un impacto mayor sobre las áreas costeras.

Costa Rica no escapa ante tal situación y, principalmente, a lo que es el aumento del nivel de los océanos (Lizano, 1997, p. 172; Lizano \& Salas, 2001, p. 172; Lizano, 2013, p. 9), debido a que al estar en un istmo y tener costas en ambas vertientes (Pacífica y Caribe) lo hace vulnerable ante futuros eventos de este tipo. Como lo indica Díaz (1999, p. 5), Costa Rica tiene más de $1100 \mathrm{~km}$ de línea de costa en el océano Pacífico y más de $200 \mathrm{~km}$ en el mar Caribe. Patrones de erosión, desde leves, moderados y hasta severos, ya se está viendo en las costas del país, como lo señala Lizano (2013, p. 10), tanto en el Pacífico como en el Caribe.

Para el caso de Costa Rica, se cuenta con el estudio efectuado por Díaz (1999), el cual determinó las zonas de riesgo ante un ascenso del nivel del mar: Punta Morales-Tárcoles. En este estudio Díaz utilizó la técnica AVVA (Análisis de Vulnerabilidad Asistido por Videograbación Aérea, propuesta por Leatherman Nicholls \& Dennis (1995), y efectuó una clasificación del frente costero de acuerdo con su vulnerabilidad ante la acción de las olas o de los cambios del nivel del mar. Combinó ese método con el reconocimiento terrestre, el estudio estereoscópico de las aerofotos, para trazar sobre ellas los límites de regresión e inundación (transgresión). Es de resaltar que ese estudio se realizó con unos pocos datos topográficos aproximados alrededor de la ciudad de Puntarenas, y en los cuales se sobrepusieron los escenarios 
del nivel del mar por calentamiento solamente. Nadie ha realizado escenarios de nivel del mar relativos a los demás componentes mareográficos, y más aún, refiriendo estos niveles al nivel " 0 " (cero) del mar o promedio de sicigias (Lizano, 2006, p. 53; 2009, p. 19).

Otro de los estudios es el de Lizano y Lizano (2010), que es una buena primera aproximación a la generación de escenarios ante el aumento del nivel del mar y otros tres componentes oceanográficas para la ciudad de Puntarenas, que se toma como referencia base. Adicionalmente, está el estudio sobre el análisis de vulnerabilidad de las zonas oceánicas y marino-costera de Costa Rica frente al cambio climático, donde se realiza un análisis a partir de datos satelitales en función de datos altimétricos obtenidos por satélite (BIOMARCC-SINAC-GIZ, 2013).

En este artículo se elaboran los escenarios de inundación por aumento del nivel del mar en dos regiones del Caribe de Costa Rica. Con ello, se pretende alertar a la población costera y a las instituciones gubernamentales sobre estos escenarios para que tomen las decisiones correspondientes ante el inminente cambio del nivel del mar. Se proponen combinaciones de nivel del mar, de tal forma que otros componentes no incluidos en este estudio, como tsunamis, puedan ser simulados también, y ayude en la toma de decisiones ante estos posibles eventos.

\section{Metodología}

El levantamiento topográfico de las zonas de Moín y Cahuita (ver Figura 1) se realizó mediante la técnica LIDAR que deriva en una gran nube de puntos para ambas zonas permitiendo obtener modelos de elevación, de alta resolución espacial del orden de $50 \mathrm{~cm}$. Estos datos son facilitados por la Comisión Nacional de Emergencia (CNE) de un sobrevuelo realizado para cubrir las costas del Pacífico y Caribe del país, entre los años 2015-2016. Tras revisar y validar los datos LIDAR de acuerdo con la clasificación internacional de la ASPRS, se procede a verificar la calidad $\mathrm{y}$, en algunos casos, se tiene que, de la nube de puntos de ambas zonas, entre un 30 y $40 \%$ de los datos de suelo se encuentran en la categoría de no clasificados, por lo que se debió proceder a la fotointerpretación y corrección de estos para contar con un área totalmente densificada que permita la obtención de cartografía a gran detalle menor a 1:1000. 
Melvin A. Lizano-Araya - Omar G. Lizano-Rodríguez

Creación de escenarios ante el aumento del nivel del mar, para las localidades de Moín y Cahuita, Limón

Costa Rica

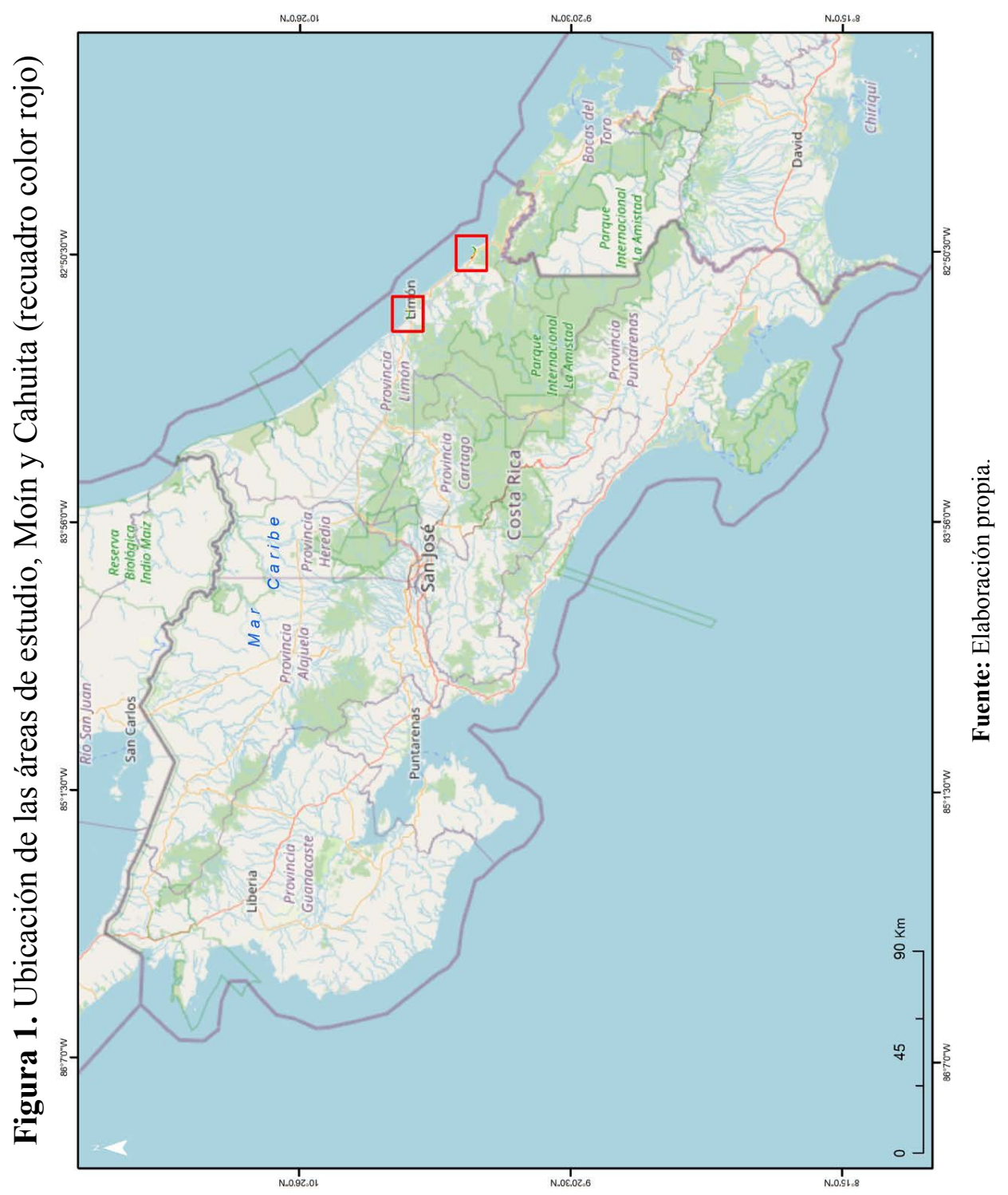


Para la zona de Moín se obtuvo, posterior a la clasificación $\mathrm{y}$ fotointerpretación, un total de 1750926 puntos de elevación; para la zona de Cahuita, 1141553. Esto permite obtener para ambos casos modelos de alta resolución espacial con un tamaño de celda de $50 \mathrm{~cm}$ como se ilustra en las figuras 2 y 3 .

Una vez que se han reclasificado los datos, se procedió a realizar el trabajo de campo para realizar las mediciones del nivel 0 de marea en función de un punto conocido (hito) de la red geodésica del IGN y, adicionalmente, se hace uso de las predicciones de marea para Limón de ese día (Lizano, 2006, p. 53). Luego se realiza el traslado a un punto terrestre conocido para con ello obtener un factor de ajuste respecto al punto de elevación, según la siguiente fórmula:

$$
\text { FC }=\text { EPM }- \text { EPCT }
$$

FC: Factor de Corrección

EPM: Elevación Punto Marea

EPCT: Elevación Punto Control Terrestre 
Al existir una carencia de un modelo Geoidal propio para el país al momento del estudio, se toma como referencia el modelo Geoidal WGS84, garantizando con ello la estandarización de toda la información sobre dicho geoide. El "datum" para elevaciones usualmente es el "datum" que define la línea de costa (Lizano, 2010, p. 19).

Con los datos obtenidos en el levantamiento de campo, en el que fueron realizadas 4 tomas de puntos utilizando un receptor Geodésico GeoMax Zenith 40, y con los datos de topográficos obtenidos del LIDAR se procedió a unificar los niveles de referencia topográficos, de manera que se tuviera en una sola capa para realizar la modelización conceptual.

El nivel "0" (cero) del mar (nivel de marea de sicigias) al que se refirieron los datos fue medido y establecido para el caso de Moín sobre el Muelle Petrolero (RECOPE) y para el caso de Cahuita sobre un espejo de agua o embarcadero hacia el oeste de la entrada principal del Parque Nacional. Es importante mencionar que los datos tomados en campo se levantan en función del nivel 0 de marea establecido en la tabla de mareas para los días y horas en que se realizó el trabajo de campo.

Se estableció un promedio entre el valor de los datos dados por el LIDAR y los obtenidos en campo del nivel de referencia " 0 " de la marea. La altura de marea obtenida a partir de los datos de campo para la zona de Moín fue de $0.37 \mathrm{~m}$, en tanto para la zona de Cahuita es de $0.51 \mathrm{~m}$. Al comparar los puntos anteriores con los dados por el LIDAR para la zona de Moín, indica $0,57 \mathrm{~m}$ y la de Cahuita de $0.69 \mathrm{~m}$. La diferencia absoluta para el primer punto es de $0.20 \mathrm{~cm}$ y para el segundo de $0.18 \mathrm{~cm}$, valor que se restó a los datos topográficos, con el fin de homologarlos al nivel " 0 " del mar, que corresponde al promedio de sicigias (Lizano, 2006, p. 53; 2009, p. 19).

Para trabajar la información de mareas astronómicas se utilizó el software para PC llamado Tidal \& Currents (Lizano, 2006, p. 53), cuyas predicciones incluidas en el programa van desde el año 1901 hasta 2099.

Los distintos niveles del mar se modelaron conceptualmente utilizando un software de Sistema de Información Geográfica (SIG), asumiendo un aumento del nivel del mar (sobre el "0" de sicigias) entre $0.36 \mathrm{~cm}$ (marea astronómica máxima en la zona) (Lizano 2006, p. 59) y aumentos de $0.72 \mathrm{~cm}, 1 \mathrm{~m}$ y $2 \mathrm{~m}$, según los criterios del informe de la CEPAL (2012, 
p. 24), como se muestra en la Tabla 1. Para el caso de estudio, al nivel promedio de las mareas máximas para el mar Caribe de Costa Rica (Lizano, 2006, p. 56) se le sumará a cada uno de los incrementos mencionados, que dicho sea de paso son conservadores, no extremos. La tasa de acenso utilizado es de alrededor de $3 \mathrm{~mm}$ por año.

Tabla 1. Aumento del Nivel del Mar para el Caribe

\begin{tabular}{|c|c|}
\hline Año & Aumento (m)* \\
\hline 2030 & 0.36 \\
\hline 2050 & 0.72 \\
\hline 2070 & 1 \\
\hline 2100 & 2 \\
\hline
\end{tabular}

Fuente: Elaborado con datos CEPAL (2012).

\section{Resultados}

La altura topográfica promedio para la zona de Moín es de $7.838 \mathrm{~m}$, en tanto que para la zona de Cahuita es de $6.688 \mathrm{~m}$, referidos al nivel " 0 " del mar, lo cual indica que tanto Moín como Cahuita son zonas sumamente vulnerables a fenómenos meteorológicos y oceanográficos. Con estos datos se encontró que la máxima altura topográfica para los primeros 500 metros a partir de la costa, para la zona de Moín, es de $38.350 \mathrm{~m}$, y la de Cahuita de $10.046 \mathrm{~m}$ a partir del nivel " 0 ". El punto en Moín se encuentre en el sector noreste a partir del Muelle Petrolero, propiamente en el área de Portete. Para el caso de Cahuita se ubica en el sector noroeste a partir del centro del núcleo poblacional de la comunidad. Las mayores elevaciones se dan en el sector noreste en Moín, así como en el noroeste en Cahuita, como se aprecia en las figuras 4 y 5 . 
Melvin A. Lizano-Araya - Omar G. Lizano-Rodríguez

Creación de escenarios ante el aumento del nivel del mar, para las localidades de Moín y Cahuita, Limón Costa Rica

Figura 4. Modelo de Elevación, Moín

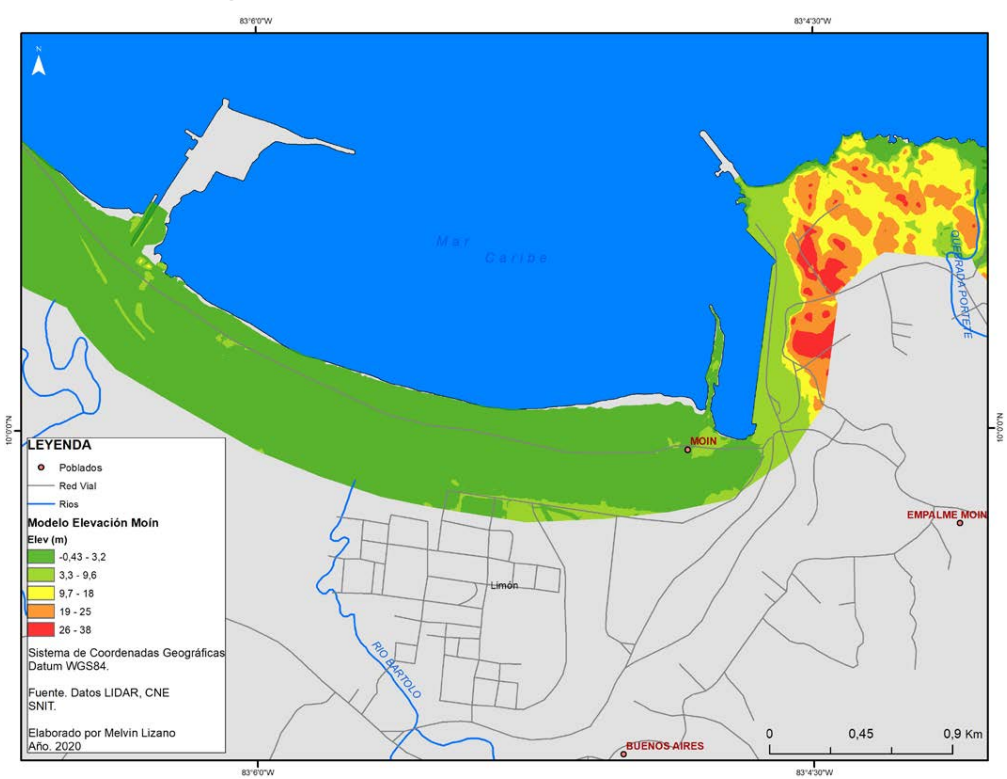

Fuente: Elaboración propia.

Figura 5. Modelo de Elevación, Cahuita

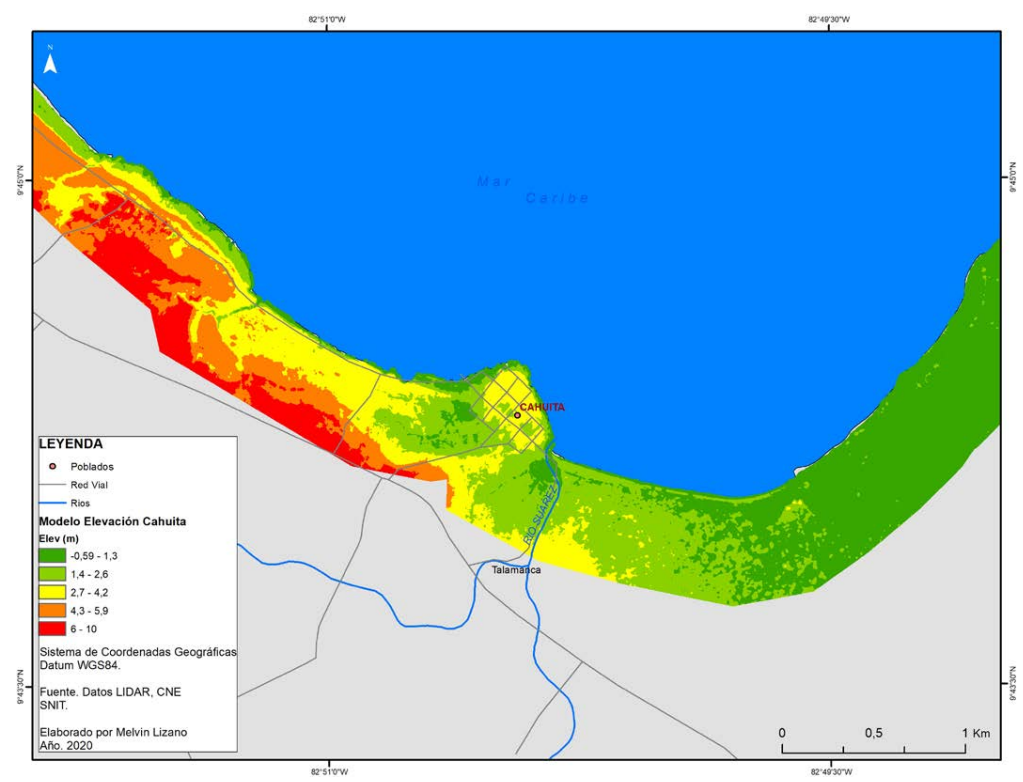

Fuente: Elaboración propia. 


\section{Mareas máximas}

Se sabe que para Cahuita una marea astronómica mayor de $0.36 \mathrm{~m}$ (Lizano, 2006, p. 59) comienza a inundar regiones del Parque Nacional Cahuita, así como en las cercanías del Muelle Petrolero en Moín, como se aprecia en las figuras 6 y 7.

Figura 6. Nivel de inundación para Moín, marea máxima promedio $0.36 \mathrm{~m}$.

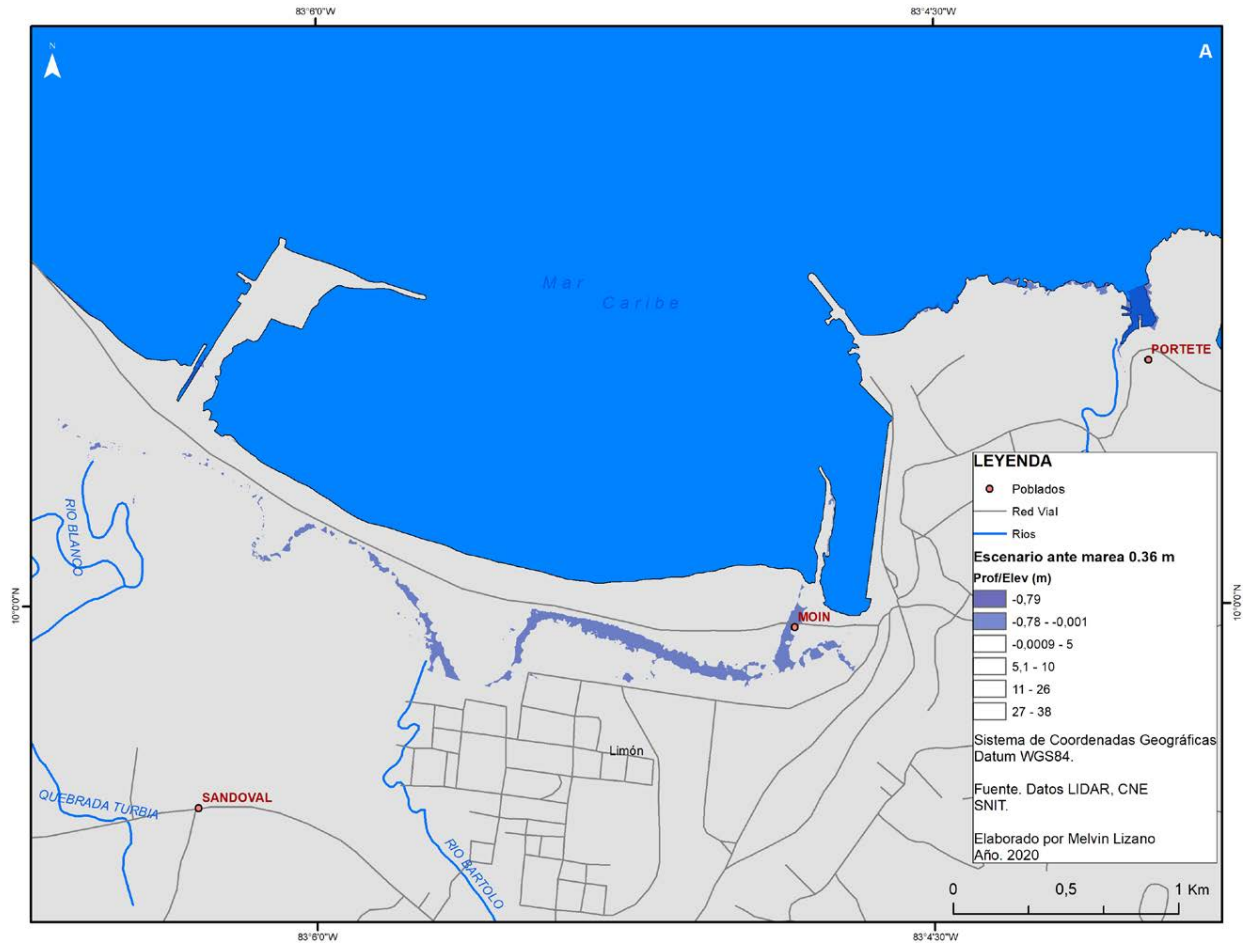

Fuente: Elaboración propia. 
Figura 7. Marea de $0.72 \mathrm{~m}$.

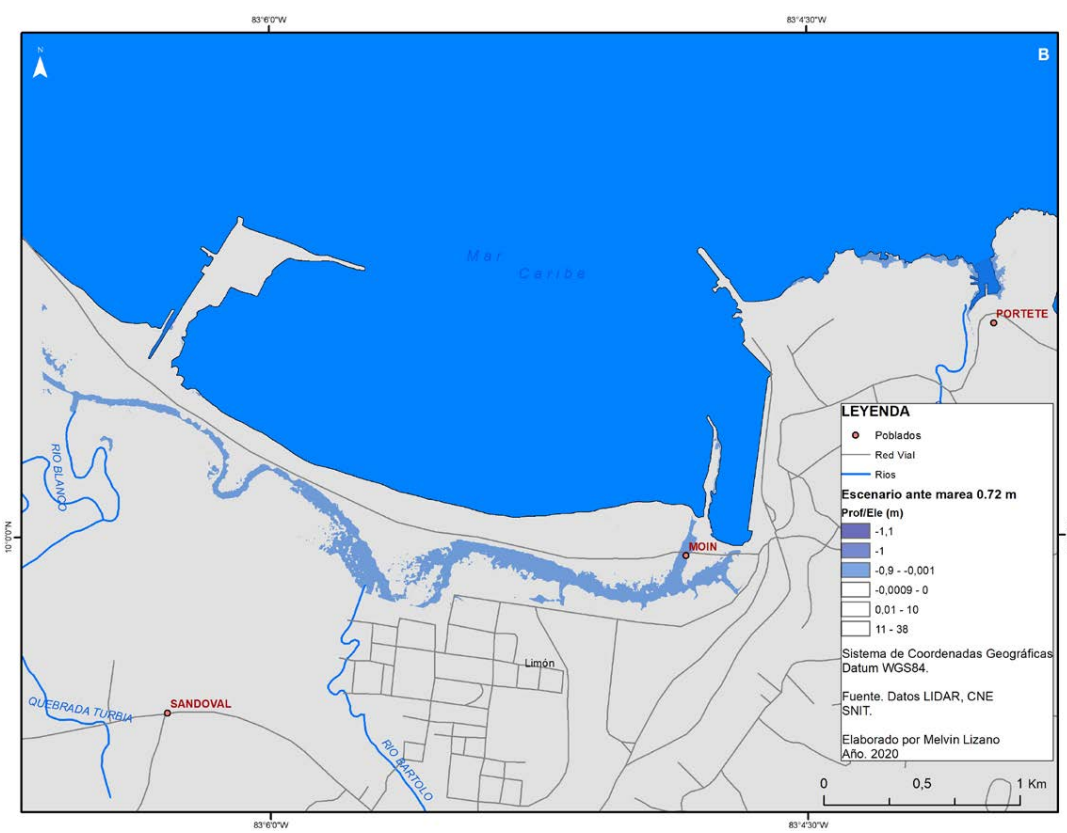

Fuente: Elaboración propia.

Múltiples escenarios se podrían construir para ambas zonas a partir de los diferentes niveles de marea astronómica: nivel " 0 ", nivel promedio ( 0.36 $\mathrm{m})$, etc., con las distintas combinaciones de los componentes mareográficos, en este caso, el aumento del nivel del mar. Sin embargo, algunos no tendrían alturas importantes que alcancen niveles de inundación para las zonas. Lo importante entonces son aquellos niveles ante el aumento del nivel del mar que tienen potencial de inundación para las zonas de estudio. Las combinaciones seleccionadas de estos niveles se resumen en la Tabla 2.

Tabla 2. Niveles mareográficos utilizados como posibles escenarios ante el aumento del nivel del mar

\begin{tabular}{|c|c|c|c|}
\hline Escenario & Año & Aumento $(\mathbf{m})$ & Marea Total* $(\mathbf{m})$ \\
\hline $\mathbf{1}$ & 2030 & 0,36 & 0,72 \\
\hline $\mathbf{2}$ & 2050 & 0,96 & 1,32 \\
\hline $\mathbf{3}$ & 2070 & 1 & 1,36 \\
\hline $\mathbf{4}$ & 2100 & 2 & 2,36 \\
\hline
\end{tabular}

$*$ Marea base $=0,36 \mathrm{~m}$. 
La marea total es la sumatoria de los valores de marea anuales más el aumento proyectado.

Las figuras 8, 9 y 10 muestran los niveles de inundación para la zona de Moín. Las observaciones que se hicieron en las zonas de estudio coinciden con los señalados en ambas figuras.

Figura 8. Niveles de inundación por mareas 1.32 m (2050), para Moín

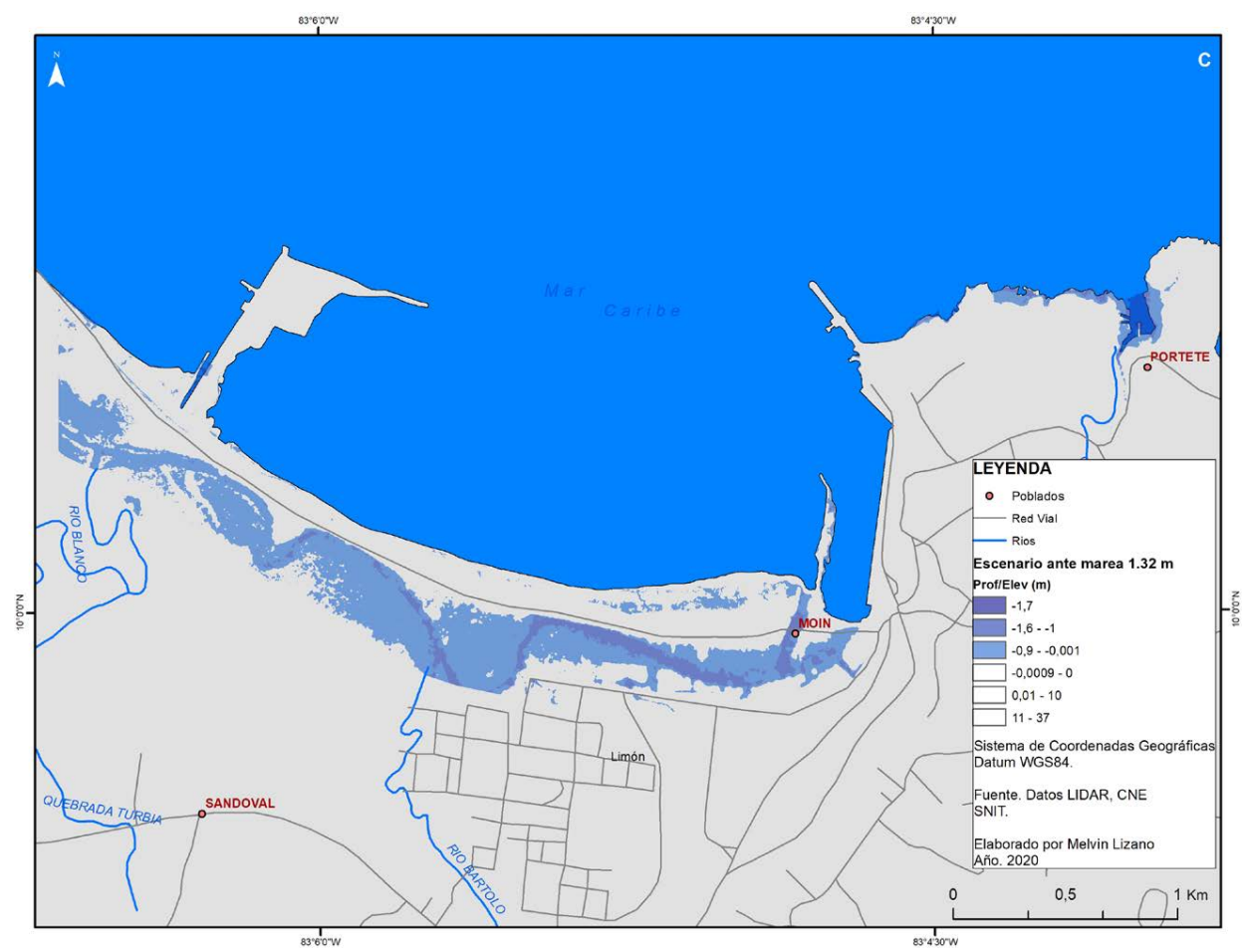

Fuente: Elaboración propia. 
Melvin A. Lizano-Araya - Omar G. Lizano-Rodríguez

Creación de escenarios ante el aumento del nivel del mar, para las localidades de Moín y Cahuita, Limón Costa Rica

Figura 9. Niveles de inundación por mareas 1.36 m (2070), para Moín

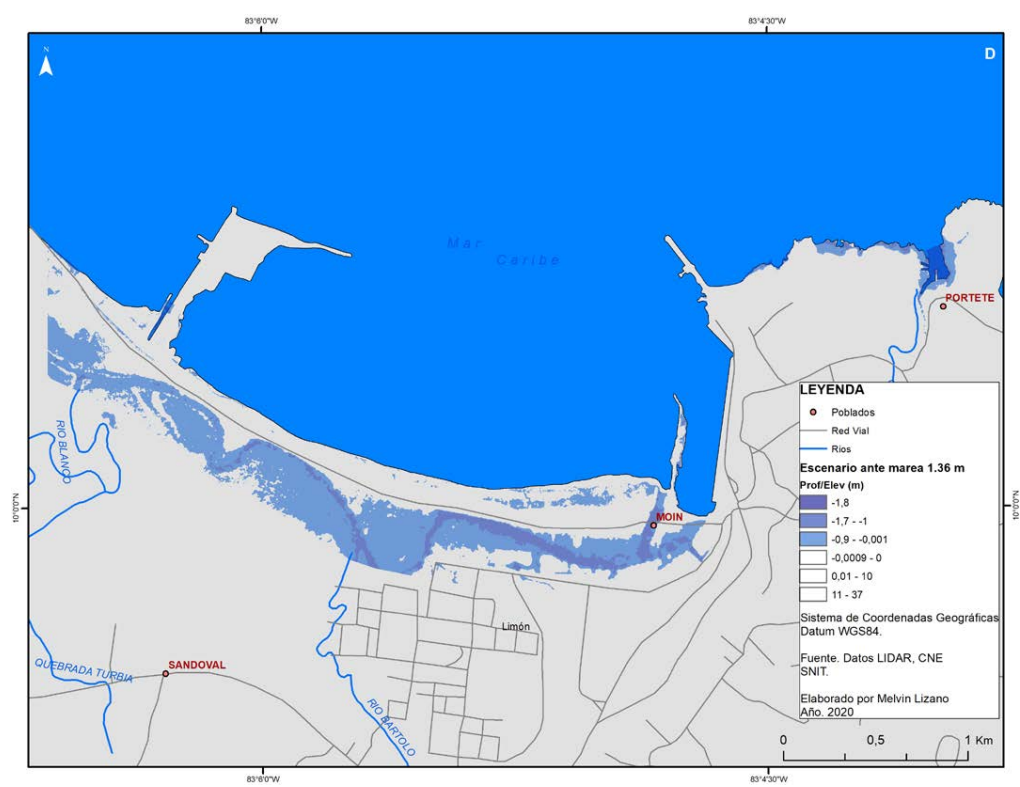

Fuente: Elaboración propia.

Figura 10. Niveles de inundación por mareas 2.36 m (2100), para Moín

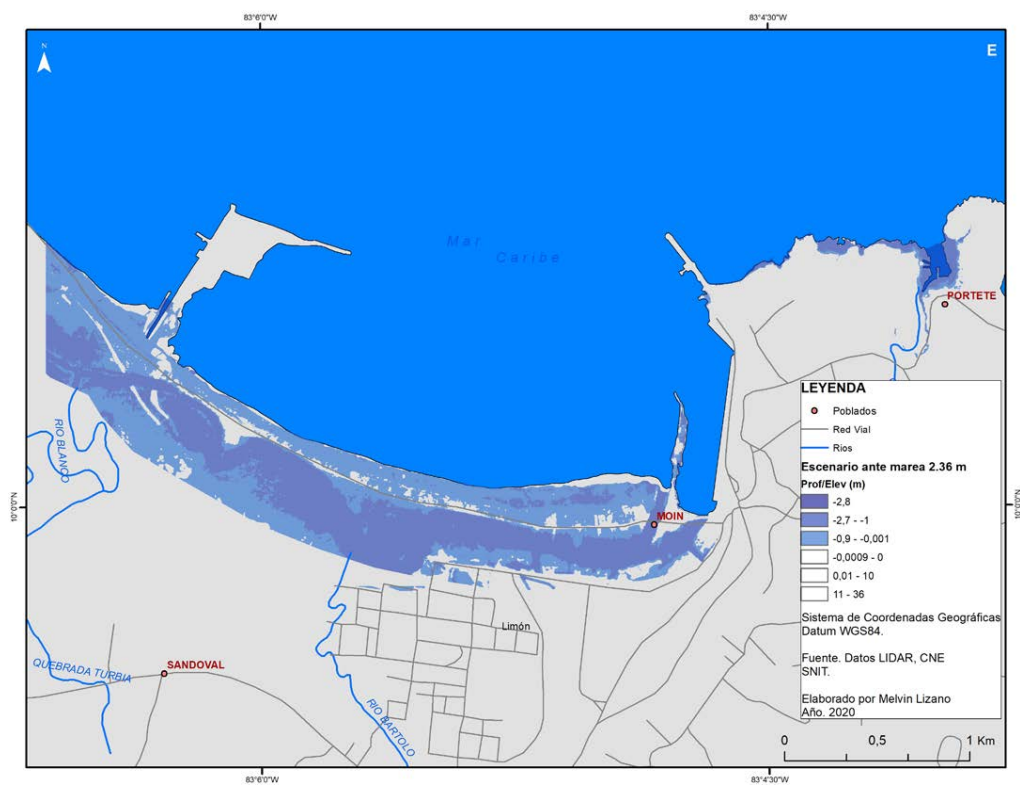

Fuente: Elaboración propia. 
Para el escenario D con niveles del mar de $1.36 \mathrm{~m}$ (para el 2070), como muestra la figura 9, el agua inunda el estero y con el escenario de $2.32 \mathrm{~m}$ (para el 2100) figura 10, prácticamente toda la zona de Moín, incluyendo los puertos o terminales de RECOPE y APM se verían afectadas.

Las figuras 11, 12 y 13 muestran los niveles de inundación para la zona de Cahuita.

Figura 11. Niveles de inundación por mareas 1.32 m (2050), Cahuita

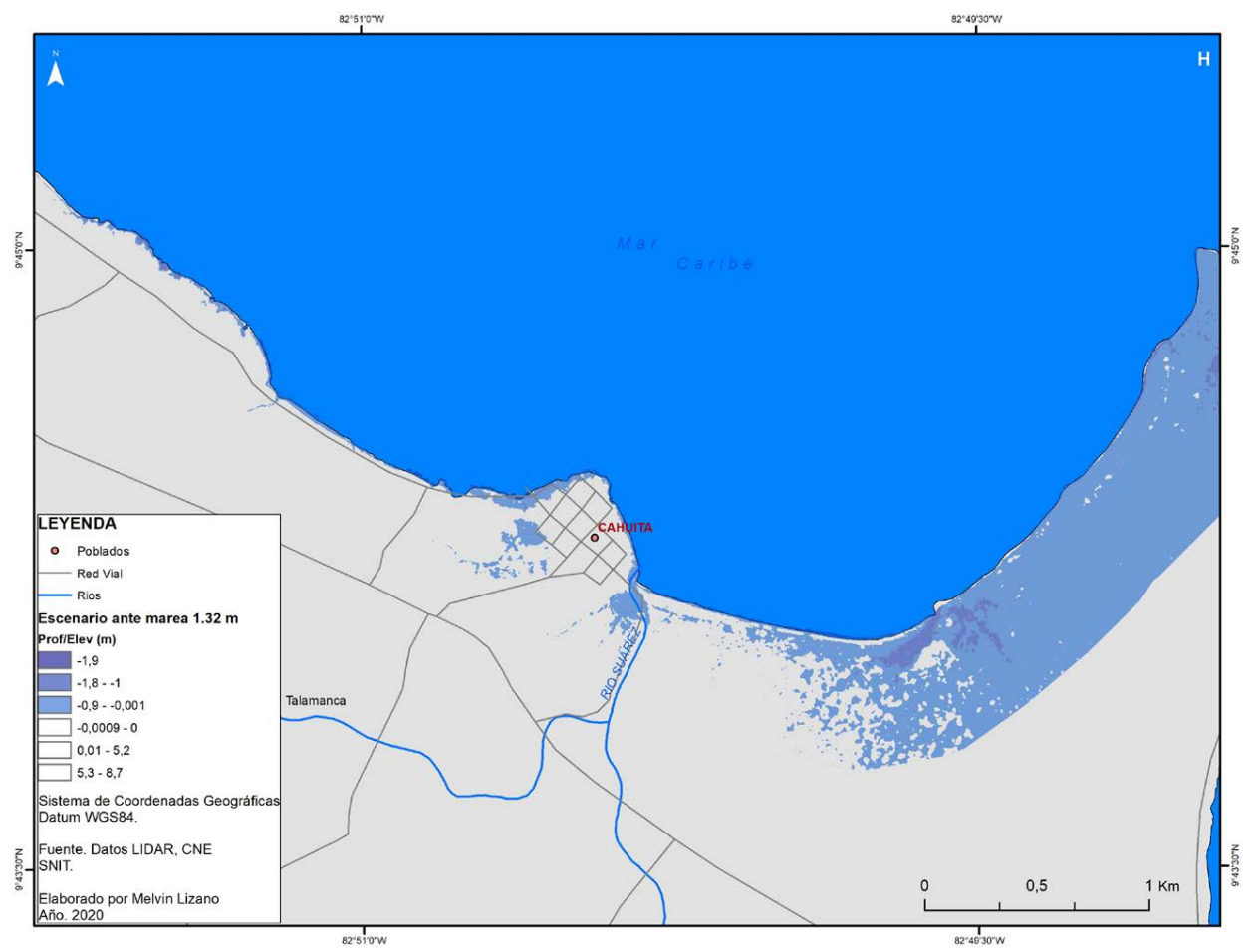

Fuente: Elaboración propia.

Las figuras 12 y 13 muestran cómo se inunda toda el área principal del Parque Nacional Cahuita con los escenarios de 1.32 y $1.36 \mathrm{~m}$ y se aísla la comunidad de Cahuita para el caso del escenario de $2.36 \mathrm{~m}$. 
Melvin A. Lizano-Araya - Omar G. Lizano-Rodríguez

Creación de escenarios ante el aumento del nivel del mar, para las localidades de Moín y Cahuita, Limón Costa Rica

Figura 12. Niveles de inundación por mareas 1.36 m (2070), Cahuita

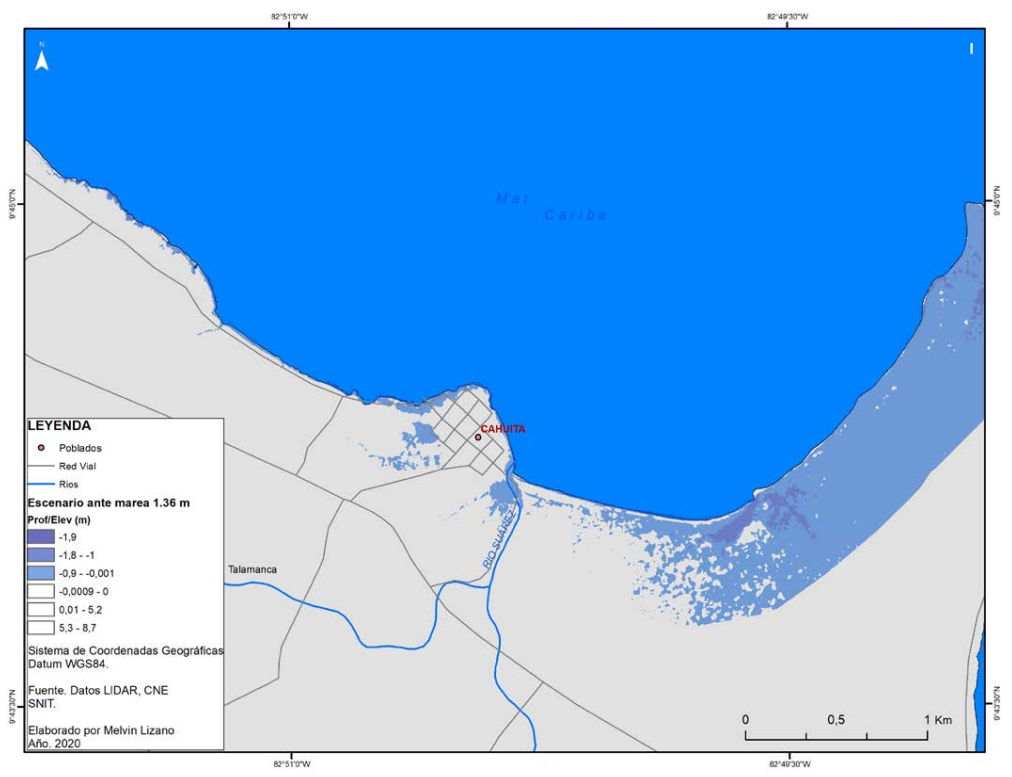

Fuente: Elaboración propia.

Figura 13. Niveles de inundación por mareas $2.36 \mathrm{~m}$ (2100), Cahuita

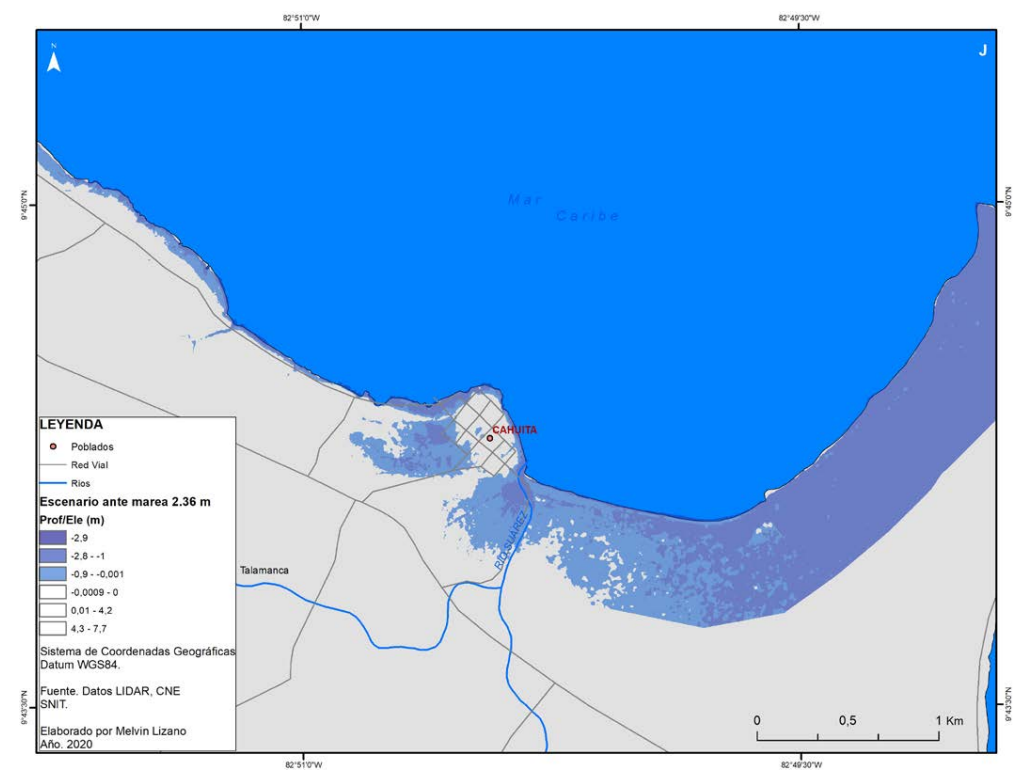

Fuente: Elaboración propia. 


\section{Discusión}

El ascenso del nivel del mar por cambio climático supondrá un problema en el corto plazo (30 años) (IPCC, 2007, p. 21) por un aumento de la frecuencia de las mareas que causan un impacto costero y su asociación con eventos hidrometeorológicos. Desde la mitad del siglo pasado el ritmo del aumento ha sido muy superior a la media de los dos milenios que le precedieron (IPCC, 2014, p. 46). Lo anterior queda reflejado en las estimaciones que muestran un ascenso significativo del nivel del mar, cuya causa directa está asociada con el aumento de la temperatura global, lo cual ha originado y está causando un derretimiento acelerado de los glaciares en muchas partes del mundo, con el consecuente problema de un mayor aporte de agua en todos los océanos (Lizano, 2013, p. 7) así como una creciente expansión térmica ligada al aumento de la temperatura de los océanos (Ibarra, Pöllänen, Álvarez, Belmonte \& Ballesteros, 2017). Lo anterior ha generado un aumento creciente del nivel del mar y se mantendrá hacia posteriores años; como se ha valorado en este documento. En consecuencia, se debe valorar la forma de prevenir eventos y desastres mayores.

Una peculiaridad obtenida a partir de los escenarios construidos es que la inundación en la zona de Moín comienza por el estero, en tanto que en Cahuita comienza por el sector del Parque Nacional donde se han venido suscitando problemas recurrentes de erosión costera y el grueso de la vegetación de la punta es hidrófila por el humedal que existe en el Parque Nacional. Ambas zonas se caracterizan por presentar las menores elevaciones, lo que provoca que, durante un ascenso del nivel de marea paulatino o extraordinario, las inundaciones se manifiesten con mayor frecuencia hacia los asentamientos que colindan con estas zonas. Como lo muestran las figuras 3 y 4 , los sectores al noreste de Moín como en el noroeste de Cahuita son las zonas que presentan las mayores elevaciones y, por ende, menos problemas ante los escenarios creados.

El ascenso del nivel es algo sumatorio, a partir del año 2090 los sectores circundantes al Parque Nacional, en el caso de Cahuita, así como en el frente de costa y en la entrada del estero por el Muelle de RECOPE, en Moín, estarán inundados a partir de mareas astronómicas o mareas altas que suceden años tras año. Posterior al año 2100 es muy factible que los sectores donde tradicionalmente se ha inundado por alguna marea extraordinaria o eventos extremos de oleajes estén completamente inundados 
bajo una capa de agua que oscilará entre los $200 \mathrm{~cm}$ o más. Esas zonas se encontrarán en Portete, Moín, así como en las áreas aledañas al centro poblacional de Cahuita y la punta del Parque Nacional.

El promedio de las mareas más altas en esta zona (Lizano, 2006, p. 59), es de $0.36 \mathrm{~m}$. En un estudio adicional de las mareas altas de 20 años de esta zona, a partir de los datos obtenidos por el software para PC llamado Tidal \& Currents (Lizano, 2006, p. 53), se demuestra que la frecuencia de superar una marea de $0.36 \mathrm{~m}$ para ambos sitios, es de 102 veces/año. Para el año 2030, con un escenario adicional de $0.36 \mathrm{~m}$, la frecuencia de tener niveles del mar con valores mayores o iguales a $0.36 \mathrm{~m}$ sería de 935.85 veces/año. Para los demás escenarios (2050, 2070 y 2100), la probabilidad de tener niveles del mar superiores a ellos será todos los días para las zonas señaladas en este trabajo.

Otro de los fenómenos que puede conllevar algunos tipos de problemas para las poblaciones cercanas sería el causado por las tormentas lejanas y/o locales (Lizano \& Lizano, 2010), que provocan el apilamiento del oleaje no modelado en este estudio (Lizano, 2007), pero que, al suceder al mismo tiempo con un período de marea alta, podría incidir en generar mayores inundaciones en zonas cercanas más allá de los escenarios aquí planteados para las zonas de Moín y Cahuita. Aunque este componente también podría producir un nivel de inundación más fuerte por la parte del estero en Moín, así como en el sector costero de Cahuita. Bajo crecientes aumentos del nivel del mar, el oleaje se constituye en el factor más dinámico y una fuente de energía suficiente para causar impacto costero en estas áreas hasta el grado de favorecer problemas de erosión más acrecentados en las zonas del estudio, aumentando los que ya tiene (Lizano, 2013, p. 19). La dinámica que produce el oleaje haría que estos sitios se inunden y modifiquen la morfología costera de forma más rápida que los escenarios pasivos mostrados en este artículo.

El panorama mostrado en este documento se puede tornar difícil si a parte del aumento del nivel del mar se consideran otras variables o fenómenos que puedan ocurrir para un día particular, como lo puede ser el apilamiento del oleaje y el fenómeno del niño por nombrar al menos dos, y que en un mismo día estos pueden llegar a mezclarse o darse todos juntos, pues implicarían aumentos importantes en el nivel del mar, al punto que podrían ocasionar inundaciones aún mayores a las mostradas en este documento. A 
partir de lo anterior, las autoridades correspondientes deberían tener algún plan de acción o de emergencia para eventos que produzcan impacto costero, o construir la infraestructura necesaria para proteger la zona y comenzar a trabajar en la posible reubicación de toda la población.

El otro componente que no se incluyó en este estudio es el aumento del nivel del mar por un tsunami. De manera que los escenarios incluidos aquí también sirven para sobreponer este componente a las condiciones de la marea existente en el momento de arribo de un tsunami, y constituye una herramienta válida para que las autoridades respectivas avalúen las condiciones del nivel del mar ante un evento de este tipo en el mar Caribe.

Adicionalmente, es importante tomar en cuenta que la variabilidad de la subida del nivel del mar y los efectos que puede tener sobre áreas concretas hacen necesarios estudios de carácter regional que tomen en cuenta los cambios a escala global (Dawahidi et al., 2019) como los reflejados en este estudio, así como otras características geográficas presentes en las áreas costeras.

Con los resultados obtenidos, cobran especial importancia los escenarios abordados en este artículo, sobre todo por el impacto que este tipo de fenómenos puede ocasionar sobre el territorio y la posible afectación de forma directa al modo de vida de los habitantes de estas zonas en términos culturales y económicos principalmente.

\section{Conclusión}

Por el grado de importancia que tiene este tema, en el cercano, mediano y largo plazo, hacia las poblaciones de las comunidades de Moín y Cahuita de la provincia de Limón, las autoridades respectivas deberían invertir en equipo tecnológico que permita cuantificar estos escenarios de manera más precisa. Se recomienda instalar un mareógrafo que permita medir y obtener patrones sobre el nivel del mar, con el fin de tener un registro continuo de esas variaciones, lo cual puede ayudar a prevenir y mitigar posibles amenazas, así como otras que puedan ocurrir y que no se encuentran citados en este documento.

En este estudio se han tratado los distintos niveles mareográficos como una superposición de niveles pasivos, sin tomar en cuenta que la dinámica de ciertos componentes como las corrientes marinas y el oleaje tienen la capacidad de modificar la geomorfología costera en un corto 
tiempo ante eventos extraordinarios. Esto puede desequilibrar el sistema para siempre antes de lo imaginable si no se toman las medidas respectivas pronto, pues, como lo señala Lizano (2013), ya el aumento del nivel del mar es una realidad en nuestras costas.

Un valor agregado del trabajo es que brindará a las autoridades de Costa Rica las herramientas necesarias para que la variable de aumento del nivel del mar sea incluida en los temas y leyes relacionados al ordenamiento territorial y la planificación urbana, así como su incorporación en los planes reguladores costeros.

Las instituciones y organizaciones nacionales que rigen estas materias o afines deben conocer este tipo de investigaciones, con el fin de que se antecedan a los escenarios acá descritos y que tengan margen de acción para mitigar posibles daños sociales, económicos y culturales de las comunidades que se pueden ver directamente afectadas por este fenómeno en un mediano y largo plazo.

Se recomienda una vigilancia permanente de las autoridades sobre el nivel del mar en estas áreas geográficas para eventualmente detectar de forma pronta cualquier aceleración en la tasa de aumento del nivel de marea y, con ello, ajustar y mejorar los modelos acá presentados.

\section{Agradecimientos}

A los estudiantes Andrés Vargas Mena y Jennifer Fernández Garro, estudiantes de Geografía de la Universidad de Costa Rica, por la colaboración en la elaboración de este artículo. A la Vicerrectoría de Investigación por apoyo a través del proyecto No. B5267.

\section{Referencias}

Bedia, J. (2004). Creación de un modelo digital de elevación para la predicción de futuros escenarios de inundación en el estuario del Río Loughor (Sur de Gales). Proyecto fin de carrera Bachelor (Honours) Environmental Science Wolverhampton University, School of Applied Sciences (U.K.).

Biodiversidad Marino Costera en Costa Rica, Desarrollo de Capacidades y Adaptación al Cambio Climático, Sistema Nacional de Áreas de Conservación \& Deutsche Gesellschaft für Internationale 
Zusammenarbeit. (BIOMARCC-SINAC-GIZ, 2013). Análisis de vulnerabilidad de las zonas oceánicas y marino-costeras de Costa Rica frente al cambio climático. San José, Costa Rica.

Comisión Económica para América Latina y el Caribe. (CEPAL, 2012). Efectos del Cambio Climático en la costa de América Latina y el Caribe: Impactos. División de Desarrollo Sostenible y Asentamientos Humanos de la CEPAL. Santiago, Chile: Naciones Unidas.

Cheng, L., Zhu, J., Abraham, J., Trenberth, K., Fasullo, J., Zhang, B., Yu, F., Wan, L., Chen, X., \& Song, Z.. (2018). Continues Record Global Ocean Warming. Advances in Atmospheric Sciences, 36(3), 249252. doi: http://doi.org/10.1007/s00376-019-8276-x

Dawahidi, T., Ibarra, D. y Gomariz, F. (2019). Estimación de la subida del nivel del mar por efecto del Cambio Climático mediante datos de mareógrafos y la serie de satélite NASA Topex-Poseidon/Jason. El caso de Valencia. Anales de Geografía de la Universidad Complutense, 39(1), 39-58. doi: http://doi.org/10.5209/AGUC.64676

Díaz, J. (1999). Determinación de las zonas de riesgo ante un ascenso del nivel del mar: Punta Morales-Tárcoles (Informe Final). San José, Costa Rica: MINAE-IMN.

Horton, B., Kopp, R., Garner, A., Hay, C., Khan, N., Roy, N. \& Shaw, T. (2018). Mapping Sea-Level Change in Time, Space, and Probability. Annual Review of Environment and Resources, 43, 482-511. doi: http://doi.org/10.1146/annurev-environ-102017-025826

Ibarra, A.D., Belmonte, F., Gomariz, F. \& Pérez, P. (2015). Evolución de la línea de costa en la Región de Murcia (1956-2013). Geo-Temas, (15), 33-36.

Ibarra-Marinas, A.D. (2016). Análisis y Evolución de las Playas de la Región de Murcia (1956-2013) (Tesis doctoral). Universidad de Murcia, España.

Ibarra, D., Pöllänen, T., Álvarez, M., Belmonte, F. \& Ballesteros, G. (2017). Estimación de futuros escenarios del nivel del mar en Valencia a través de datos de mareógrafos. XXV Congreso de la Age. Naturaleza, territorio y ciudad en un mundo global. 647-654.

Intergovernmental Panel on Climate Change. (IPCC, 2007). Cambio climático 2007: Informe de síntesis. Informe del Grupo Intergubernamental 
de Expertos sobre el Cambio Climático. R. Pachauri \& L. Meyer (Directores). Ginebra, Suiza: IPCC.

Intergovernmental Panel on Climate Change. (IPCC, 2014). Cambio climático 2014: Informe de síntesis. Contribución de los Grupos de trabajo I, II y III al Quinto Informe de Evaluación del Grupo Intergubernamental de Expertos sobre el Cambio Climático. R.K. Pachauri \& L.A. Meyer (Eds.). Ginebra, Suiza: IPCC.

Lambrechts, C. (2007). Global Outlook for Ice \& Snor. H. Main. (Directora), -Arendal, Noruega: UNEP/GRID.

Leatherman, S., Nicholls, R. \& Dennis, K. (1995). Aerial Videotape-Assisted Vulnerability Analysis: A Cost-Effective Approach to Asses Sea-Level Rise Impacts. Journal of Coastal Research. Special Issue, (14), 15-25.

Lizano A.M. \& Lizano R.O. (2010). Creación de escenarios de inundación en la Ciudad de Puntarenas ante el aumento del nivel del mar. InterSedes, XI(21), 215-229.

Lizano, O.G. (1997). Las Mareas Extraordinarias de 1997 en la Costa del Pacífico de Costa Rica. Top. Meteor. Oceanogr., 4(2), 169-179.

Lizano, O.G. \& Salas, S. (2001). Variaciones geomorfológicas en los últimos 50 años de la Isla Damas, Quepos, Costa Rica. Rev. Biol. Trop., 49(2), 171-177.

Lizano, O.G. (2006). Algunas Características de las Mareas en la Costa Pacífica y Caribe de Centroamérica. Ciencia y Tecnología, 24(1), 51-64.

Lizano, O.G. (2007). Climatología del viento y el oleaje frente a las costas de Costa Rica. Ciencia y Tecnología, 25(1 y 2), 43-56.

Lizano, O.G. (2009). Batimetría, modelo de elevación digital y sus aplicaciones. Revista Azimuth, 4(10): 18-21.

Lizano, O. (2010). Batimetría, modelo de elevación digital y sus aplicaciones. Revista AZIMUTH 10 DIGITAL, 18-21.

Lizano, O.G. (2013). Erosión en las playas de Costa Rica, incluyendo la Isla del Coco. InterSedes, 14(27), 6-27. doi: http://doi.org/10.15517/ ISUCR.V14I27.10405

Mcinnes, K., Walsh, K., Hubbert, G. \& Beer, T. (2003). Impact of Sealevel Rise and Storm Surges on a Coastal Community. Natural Hazards, (30), 187-207. doi: http://doi.org/10.1023/A:1026118417752 
Neremet, R. S., Beckley, B. D., Fasullo, J. T., Hamlington, B. Masters, D. \& Mitchum G. T. (2018). Climate-change-driven accelerated sea-level rise detected in the altimeter era. Proceedings of the National Academy of Sciences, 115(9), 2022-2025. doi: 10.1073/ pnas. 1717312115

Otto-Bliesner, J., Marshal, T.S., Overpeck, I.J., Miller, G.H., Hu, A. \& CAPE Last Interglacial Project members. (2006). Simulating Arctic Climate Warmth and Icefield Retreat in the Last Interglaciation. Science, 311(5768), 1751-1753. doi: http://doi.org/10.1126/ science. 1120808

Programa de las Naciones Unidas para el Medio Ambiente. (PNUMA, 2013). GEO-5 Perspectivas del Medio Ambiente Mundial. Medio ambiente para el futuro que queremos. Programa de las Naciones Unidas para el Medio Ambiente.

Tarolli, P., Cao, W., Sofia, G., Evans, D. \& Ellis, E. (2019). From features to fingerprints: A general diagnostic framework for anthropogenic geomorphology. Progress in Physical Geography, 43(1), 95-128. doi: http://doi.org/10.1177/0309133318825284

Van der Meulen, F., Witter, J.V. \& Marens, S.M. (1991). The use of a GIS in Assessing the Impacts of Sea-Level Rise on Nature Conservation along the Dutch Coast. Landscape Ecology, 6(1/2), 105-113.

Wright, L., Syvitski, J. \& Nichols, C.R. (2019). Sea Level Rise: Recent Trends and Future Projections. En L. Wright \& C. Nichols (Eds.), Tomorrow's Coasts: Complex and Impermanent. Coastal Research. Springer, Cham. Library, vol 27. 